\title{
DETERMINANTS OF INDUSTRY 4.0 TECHNOLOGY ADAPTION AND HUMAN - ROBOT COLLABORATION
}

\author{
Martina PORUBČINOVÁ ${ }^{1}$, Helena FIDLEROVÁ ${ }^{2}$ \\ ${ }^{1}$ SLOVAK ACADEMY OF SCIENCES IN BRATISLAVA, CENTRE OF SOCIAL AND PSYCHOLOGICAL \\ SCIENCES SAS, ŠANCOVÁ 56, 81105 BRATISLAVA, SLOVAKIA \\ ${ }^{2}$ SLOVAK UNIVERSITY OF TECHNOLOGY IN BRATISLAVA, FACULTY OF MATERIALS SCIENCE AND \\ TECHNOLOGY IN TRNAVA, INSTITUTE OF INDUSTRIAL ENGINEERING AND MANAGEMENT, \\ ULICA JÁNA BOTTU 2781/25, 91724 TRNAVA, SLOVAKIA \\ martina.porubcinova@savba.sk, helena.fidlerova@stuba.sk \\ Received 8 April 2020, accepted 03 June 2020, publisher 25 June 2020
}

\begin{abstract}
Aim of the paper is to analyse specific factors of human-robot collaboration and adoption to Industry 4.0 technologies in relation to working in mixed teams and in virtual work environment. Based on literature review and current findings in unified theory of technology acceptance and use of technology (UTAUT) model and its extensions, new endogenous factors might be included/considered, in a UTAUT model, namely emotional attitude (trust towards technology, perceived threat) and new moderators such as a location of organization and type of workplace-based training in organization. Team identification and self-extension process are intended as drivers for development of positive attitudes in human-robot collaboration and prerequisites of sustainable human-robot interaction. Regarding the findings in human-robot collaboration, the authors suggest including the type of personality and the attitudes towards Human-Robot collaboration among the extended factors of technology adoption within Industry 4.0 strategy. Emotional attachment acts as a relevant factor of successful HumanRobot collaboration that can be maintained by a support of team identification and selfextension process. For the development of positive attitudes in human-robot collaboration, principles of personalized design can be applied, e. g. engaging the workers in designing specific features of the robot. It can be supposed that specific models of technology adoption as UTAUT/UTAUT2 will require new extensions in a context of work analysis in a virtual work environment, e.g. health and psychological assumptionsIn the paper, subjective assessment of the increase in well-being of the workers after the adoption of Industry 4.0 technologies is identified as one of the new Industry 4.0 outcomes within the human capital definition of Industry 4.0.
\end{abstract}

\section{Key words}

Human-robot collaboration, attitude, adaption of technology, UTAUT model, strategy Industry 4.0, sustainable human-robot interaction 


\section{INTRODUCTION}

The human capital factor is an aspect of crucial importance for the correct implementation of Industry 4.0. Even if Smart Factories are expected to be equipped with a higher level of automation, "this significant innovation does not entirely eliminate the need for the human operators - on the contrary - it requires them to collaborate with the robots and execute hybrid tasks" [1]. The main benefits of using human robot collaboration in a shared setup are the advantages focused on the reliability of the robots with the flexibility of the humans. Safety and sustainability issues, along with the attitudes towards Human-Robot collaboration, protection of health, willingness to cooperate within Human-Robot work teams, need to be considered comprehensively when analysing the creation of a shared information base within the Industry 4.0 concept. The well-being of workers is the prerequisite of a successful and sustainable process of the Industry 4.0 adoption.

It is been decades since the experts have started to examine the types of interactions that are needed for desirable interchanges between robots and humans [2]. Thus, creations of human-robot teams that are efficient and effective and take advantage of the skills of each team member have become one of the goals of the IT system management theories. From a managerial point of view, Human-Robot interaction differs from already known humancomputer and human-machine interactions as "it concerns with complex, dynamic control systems, exhibit autonomy, and cognition and which operate in changing real-world environment" [2].

Human-centred design in Human-Robot interactions that looks beyond technology issues can concentrate not only on safety issues. (so not to harm) IT professionals and human factors practitioners in multidisciplinary cooperation can focus on optimizing the technology adaption process (in technology adaption intentions and technology adaption behaviour analysis) as well as on developing the skills that are important and beneficial for working in human-robot hybrid teams.

Within Industry 4.0 adoption, there are not only digital skills examined as a part of hybrid work competencies owing to emerging of hybrid work tasks. Specific human competencies that create a comparative advantage, e.g. as a mind-set, orientation, openness, skills of management are crucial for the transmitting of business ideas to practice.

Currently, we are witnessing an emerging emphasis on the analysis of sustainability factors of the Industry 4.0 adoption. As stated by Sousa [3], there are several critical success factors of introducing Industry 4.0 beyond the digital skills related to the environmental and social sustainability that can be identified recently, like communication, management leadership, training and capacity building, empowerment, teamwork, organizational culture, national culture, and regional differences.

\section{INDUSTRY 4.0 CONCEPT IN HUMAN CAPITAL PERSPECTIVE}

As we suppose, the essence of the Industry 4.0 innovation is the interconnection of individual technology components and human workers communicating with each other at an unprecedented rate. The essence of Industry 4.0 concept is the introduction of network-linked intelligent systems, which perform self-regulating production: people, machines, equipment, and products will communicate to one another [4]. As author [5] stated, Industry 4.0 requires answers and solutions for different topics: man, and labour, business and strategy examples, how to handle the data-overflow, cyber-security, standard and interoperability, medium sized businesses and users, etc. 
To avoid technological determinism and to emphasize the interrelationship between technological development and changes in work competences, working culture and social innovations in the context of Industry 4.0, it is necessary to move the definition of Industry 4.0 towards a comprehensive socio-technical definition of the process to capture sustainability of the process in its social dimension. This approach - in addition to using the human capital factor as an essential indicator of the Industry 4.0 formation - allows capturing the benefits and challenges as well as the difficulties and threats associated with Industry 4.0 in its social dimension [7]. Therefore, the Industry 4.0 definition based on a human capital approach can be formulated as: "Innovation in manufacturing, based on increasing the communication flow and communication capabilities of $H-H$ and $H-R$ collaboration of all production entities to achieve flexible and customer-oriented production, coupled with human capital transformations".

In the frame of the Industry 4.0 human capital definition, the attention is focused on sustainable development of the Industry 4.0 principles, e.g. in the field of the specific requirements analysis created by Industry 4.0 concerning the age and gender factors. Based on information from literature analysis focused on human capital, the issue to find how to promote enjoyable interaction between individuals and their robots belongs to the key challenges addressed by the innovation process in production and IT systems.

As stated by Richert at al. [6], new forms of artificial intelligence on the one hand, and the ubiquitous networking "everything with everything" on the other hand, that characterize the $4^{\text {th }}$ industrial revolution result in a changed understanding of human-machine interaction. However, understanding the effective teamwork in human-robot collaboration, including attitudes and emotional attachment towards human-robot collaboration in hybrid work teams belong to both, an important and much-understudied areas in the IS literature [8].

The shift towards examining the design features of technologies and tools that support the wellbeing of Human-Robot collaboration can be identified within the current analyses. Such a focus can bring the impulse for support of women's and men's wellbeing in the work process to the future. We believe that human resource optic is needed to be applied in the Industry 4.0 analysis to emphasize the changes in human capital that can be seen in connection with the sustainable development and adaptation of Industry 4.0.

\section{PSYCHOLOGICAL FACTORS OF HUMAN- ROBOT COLLABORATION}

We agree with Loenhard [9] that human happiness and well-being are at the heart of the decision making in every situation. Human-centred approach and accentuation of the well-being of human workers in hybrid work teams (or mixed teams) within Human-Robot collaboration covers are crucial and mean more than mere areas such as safety of the workers, attitudes towards Human -Robot collaboration (trust, threats), willingness to cooperate within Humanrobot work teams, and protection of health.

The era of robotics began with a human performance need: how to manipulate highly radioactive objects without exposing the human operator [10]. According to Peters et al. [11], there are many opportunities within various spheres for technologies to be autonomy-supportive and research shows that making them so will foster engagement, motivation, and wellbeing (of human workers). Although several models of developing a long term flourishing experience of working with technologies as Positive Computing, Positive Design or Positive Technologies have been introduced, "clear design features relating to wellbeing determinants, pleasure, virtue or meaning have yet to be identified" [11]. Growing understanding of the key psychological needs that promote substantial motivation and well-being (feeling the autonomy as acting following one's goals and values as well as competence and relatedness to the others) lead to the changes in the field of engineering, shifting the attention to designing autonomous systems 
that place human autonomy to the centre of interest. Such a human-autonomy supportive technology design approach is represented by the devices that offer options and choices to users, or building design according to user's preferences or several options in modes of communication and interface, and active designing can help to raise the autonomy of users and their wellbeing.

As stated by scholars, recently, we know very little what makes such (human-robot) teams successful. Researchers suggest that e.g. the exploration of emotional attachment between teams and their technologies could be vital to understanding of how to facilitate better teamwork in teams. Studies of the emotional attachment of humans towards robots as co-workers as a band or a connection between individuals and their technology. When people are emotionally attached to their technology, they become more engaged in using the technology, and enjoy the interaction with it more [12].

New modes of production in hybrid human-machine collaboration bring the need for examination of psychological factors of human-robot interaction. In this context, robots are defined as technologies with virtual and physical embodied actions, that - especially in a case of Embodied Physical Action robots (EPA) robots - can lead individuals to strong emotional responses $[12,13]$. Following the reactions of different personality types on the different (industrial, mechanic) design of social robots is one of the fertile and relevant ways of Industry 4.0 research. Working with robots without usual safety areas requires to follow how (and to what degree) a specific design and integration of natural user interfaces could enhance the acceptance of humanoid robotic design in hybrid working teams. Different production environments (industry, service) seem to be connected with different robotic design, as stated by Richert et al., [6], as well as different types of personalities are correlated with different types of reaction to the robot as a working partner.

When comparing the humanoid and robotic design of robot in the context of different human reactions within the human-robot collaboration, empirical results have confirmed more stable reaction of different personality types to humanoid robot (false, mistaken) reactions, while the reactions to robots false and mistaken behaviour have been found depending on the type of personality. Different personality types, as well as the attitudes towards human-robot collaboration, seem to act as important factors for developing successful human-robot collaboration.

As the attitudes towards technology are being broadly examined to follow emotional attachment towards technology, various consequences, e. g. feelings of workers' satisfaction, effectiveness and performance are being reflected in several models of adoption of a collaborative system. Models of effective adoption of Collaborative Systems that explain the user acceptance of information technology could be applied in the context of effective introducing and development of Industry 4.0 concept, as the unified theory of technology acceptance and use of technology (further UTAUT). UTAUT, an integrated model of eight theories which include the Theory of Reasoned Action (TRA), Technology Acceptance Model (TAM), Motivational Model (MM), Theory of Planned Behaviour (TPB), Combined TAMTPB (C-TAM-TPB), Model of PC Utilization (MPCU), Innovation Diffusion Theory (IDT) and Social Cognitive Theory (SCT), was formulated with four core determinants of interaction and usage -performance expectancy, effort expectancy, social influence, and facilitating condition- and up to four core determinants of key relationships - gender, age, experience, voluntariness of use [14].

\section{UTAUT MODEL AND ITS EXTENSION}

The UTAUT model contains four essential determining components and four moderators. According to the model, the four determining components of behavioural intention and usage 
behaviour are performance expectancy, effort expectancy, social influence and facilitating conditions [6]. Gender, age, experience, and willingness to use are the moderators that affect the usage of technology (see Figure 1).

The determining components are defined as follows by Venkatesh et al [14]:

Performance expectancy is defined as the degree to which an individual believes that using the system will help him or her to attain gains in job performance. In looking at the gender and age effects, it can be expected that the influence of performance expectancy on behavioural intention will be moderated by gender and age, such that the effect will be stronger for men and particularly for younger men.

Effort expectancy is defined as the degree of ease associated with the use of the system. Based on empirical testing, the influence of effort expectancy on behavioural intention can be expected to be moderated by gender, age, and experience, such that the effect will be stronger for women, particularly younger women, and particularly at early stages of experience.).

Social influence is defined as the degree to which an individual perceives that important others believe he or she should use the new system. According to the UTAUT model, the influence of social influence on behavioural intention can be expected to be moderated by gender, age, voluntariness, and experience.

Facilitating conditions are defined as the degree to which an individual believes that an organizational and technical infrastructure exists to support the use of the system. Even if the issues related to facilitating conditions are largely captured within the effort expectancy construct, the effect is expected to increase with experience as users of technology and the age. Facilitating conditions are the factor significant in the case of older workers, particularly with increasing experience.

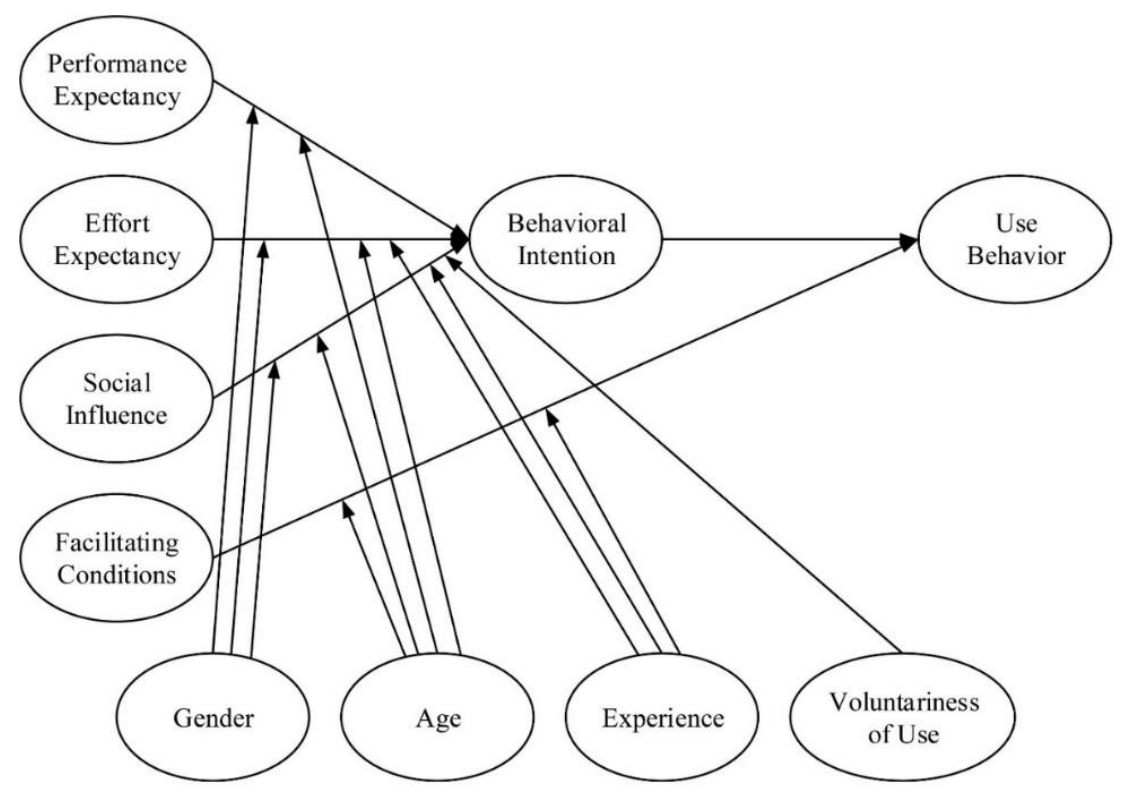

Figure 1 UTAUT model introduced by Venkatesh [13]

Organizational psychologists have noted that older workers attach more importance to receiving help and assistance on the job. This is further underscored in the context of the complex IT use, given the increasing cognitive and physical limitations associated with age. These arguments are in line with empirical evidence from Morris and Venkatesh [15]. Thus, 
when moderated by experience and age, facilitating conditions will have a significant influence on user behaviour.

The attitudes toward using technology, self-efficacy, and anxiety are theorized not to be direct determinants of intention in the UTAUT model. However, in its extension, the UTAUT 2 model [14], several new determinants of the technology interaction and the users have been included, such as price, value, trust and hedonic motivation. The key determinants are modified also by new moderators within the new contexts that include location and type of organization. Moderators are examined in relation to the behaviour intentions to use technology in organization context (within determinants of performance expectancy, effort expectancy and social influence) and in the relation to technology use (within conditions factor).

Based on literature review of the UTAUT model extensions, new endogenous factors might be included in a model of technology adaption and use, namely emotional attitude (trust towards technology, perceived threat) and new moderators as a location of organization and type of workplace-based training in organization (Fig. 2). Also, not many studies have paid attention to the new types of outcomes of the Industry 4.0 technology adaption within the UTAUT or UTAUT 2 models. Subjective assessment of workers wellbeing after the adoption of Industry 4.0 technologies can be identified as one of the new Industry 4.0 outcomes within the Industry 4.0 human capital definition.

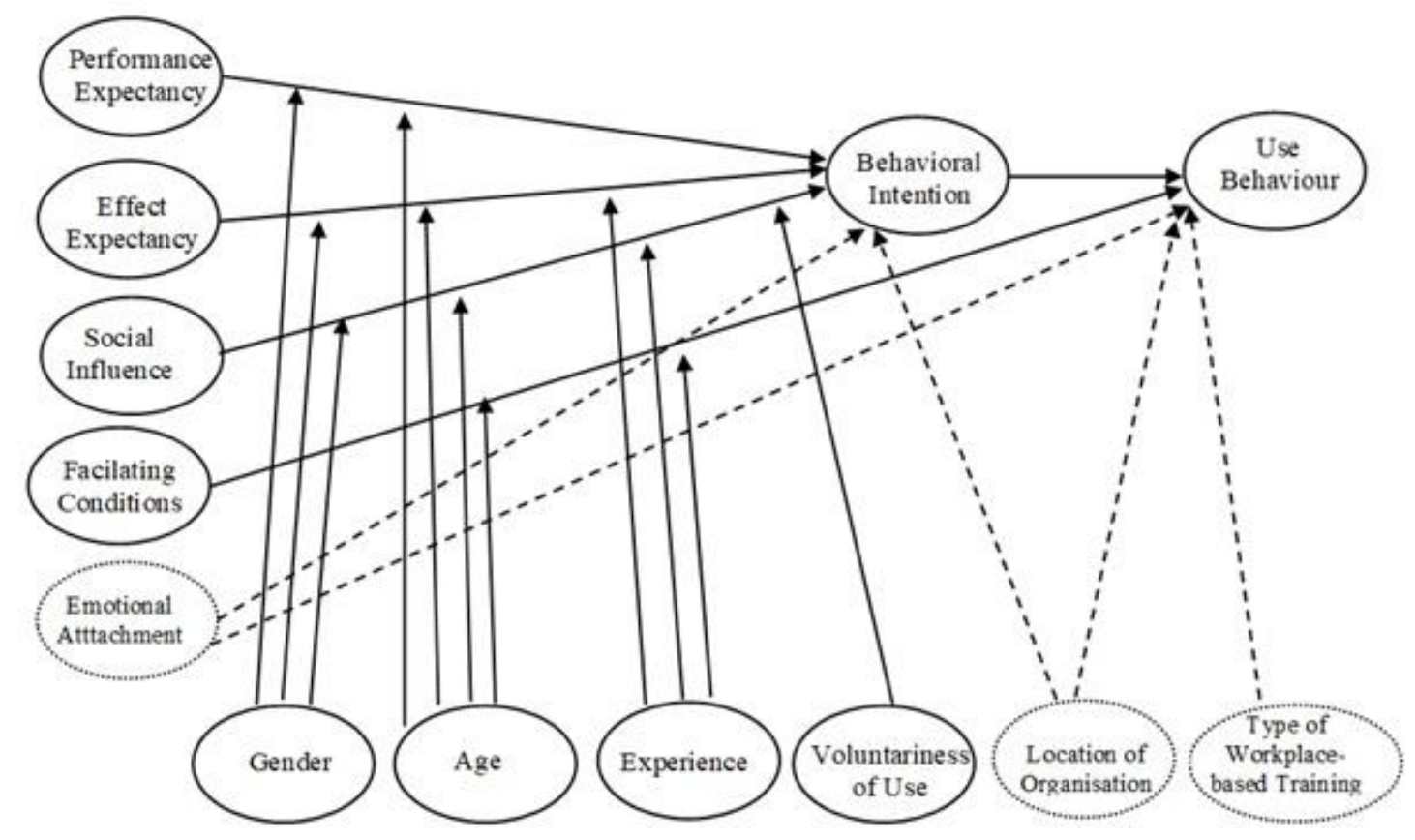

Figure 2 UTAUT model modified within Industry 4.0 human capital definition (drawn by authors)

Several concepts in a frame of worker's wellbeing within the Industry 4.0 development point to the importance of attitudes and emotional relationship to co-bots within human-robot mixed work teams. For example, the concept of self-extension as a powerful mechanism of augmenting one's identity to external objects has been recently used to represent identification with digital goods and services.

According to the IT identity theory, the individual can become identified with information technology by including it to their self-concept. For example, participatory design as a collaborative design approach involving all stakeholders allows robots to reflect the team's 
needs and attitudes, which should help teams develop emotional bonds with the robots [16]. Emotional attachment can be developed in a self-extension concept through creating and EPA robots by a worker or providing feedback during the process of planning hoe to introduce new technology in a firm [6].

Not only self-extension acts as a mechanism of transmitting the emotional attachment towards technology but also the team identification in the sense of the high value of team membership. Team identification can act as a way of developing of the emotional attachment of a team towards technology that is issued by the team:" teams that are highly identified should be more emotionally attached to their robots", as they are more likely to see EPA robots as a representation of a team.

In the context of the METUX model, which examines the factors of adoption of new technologies, authors predict that "users will be likely to adopt new technology to the extent that they are autonomously motivated to do so". According to the authors, person's willingness to adopt a technology would be influenced by anticipated competence to use it, which can be also framed as perceived easy-of-use, that can be influenced by aesthetics-usability effect marketing, user's prior experience and general attitude toward technology. Finally, in the METUX model that adopts a human-autonomous-centered approach in designing HumanComputer interaction, also the factor of "societal experience" that covers the experience of all members of a society beyond the users of the technology is being analyzed [10].

The UTAUT model has been adopted in research technology adaption not only for individuals, but also for groups and organizations in different environments. The model offers a tool for the managers' optimal decision making, formulating the strategy and to assess the successful new technology introductions. It helps them to recognize the drivers of acceptance to proactively design interventions and identify the strategy targeted at the users that may be less inclined to adopt and use new technologies.

The practical implication of psychological factors identification regarding worker's experience with technology lies in improving the implementation process of new technologies. Thus, in the context of sustainable strategy Industry 4.0, attitudes towards cooperating robots can be tested as the factor of influence, extending the optics of the UTAUT model towards the UTAUT model extension, including user technological acceptance.

For managers, it is important to follow the DMAIC (define-measure-analyze-identify and control) model. In the first step, strategy for successful technology adaption, objectives in long and short term and factors, chosen as most significant regarding chosen strategy and objectives, should be defined. Then data are measured about the process indicators for the key factors, data will be analysed and used as baseline for identification of potential for improvement. The final step is control procedure if stated objectives and indicators were achieved, constrains eliminated and possibilities for future improvement identified.

Concerning psychological, societal and organizational factors of adapting to new technologies, we suppose that designing and implementing technologies with the emphasis on wellbeing impact can be identified as one of the future challenges of the sustainable Industry 4.0 strategy. The important findings of emotional attachment towards technology in humanrobot collaboration confirm that emotional attachment towards technology used in a team supports the performance and viability of a team.

On an individual level, testing the level of motivation (autonomous, controlled within METUX model) and human wellbeing in the adoption of new technologies can enrich the technology implementation. 


\section{CHALLENGES IN MIXED WORK TEAMS}

As the authors point, bringing together humans and robots is not as trivial as it may sound [15]. A hazardous situation may arise while robots and machines operate in a relatively large workspace, also even in the case where the robotic arms operate in a completely pre-defined way, the unpredictability of human behaviour may still result in dangerous interaction. Collaboration schemes that can guarantee the safety of human operators have been captured in standards that regulate the interaction between humans and automation (ISO 10218 Part 1 "Safety of Robots", Part 2 "Safety of Robot Integration). ISO/TS 15066 "Robots and robotic devices - Collaboration Robots include guidelines for collaborative robot operations with humans in a shared workplace: a) established minimum separate distance, b) established a maximum safe speed, c) tracking operator position d) determining and avoiding potential contact, e) avoiding potential collision, power and force limiting, ergonomic, medical requirements and so forth.

Under this context, hybrid production operations are classified into shared tasks and workspace, b) common tasks and workplaces and c) common tasks with a separate workspace. In these operations there are several interaction methods means for coordinating the activities of humans and robots, that can be divided into direct physical interaction and remote contactless interaction. Direct physical interaction includes robot assistants as the directly interacting working partners, collaboration robots that introduce mechanical devices that provide guidance using servomotors while a human operator provides motive power, portable robots, power amplifying assistance devices, BAXTER robots with tactile feedback. Specific new robotic products include lightweight robotic arms, sensory equipment (sensors, cameras), safety equipment for the preservation of the human operator's health including laser scanners and safety curtains).

Hybrid work tasks, resulting in emerging hybrid work competencies raise the meaning of digital competencies and positive attitudes towards human-robot collaboration. Engineers declare the main challenges human-robot collaboration recently even in the field of a more dynamic monitoring approach, as "the current status of the robot is presented usually in a format that is not designed for non-expert operators ( e.g. robotic code). The aim is declared in increasing the awareness of the robot's operation status, underlying safety functions to increase the feeling of comfort and acceptance of the operators when working with such powerful equipment". There are several specific technologies for achieving human-robot collaboration, as force sensors, human-machine interfaces, cameras, and tools that can be applied instead of button communication, as smartwatch interface, augmented reality glasses.

Within the Industry 4.0 concept, depended on information exchange in human-robot collaboration, these innovative tools bring intuitive and easy to see applications to communicate. Augmented reality with its benefits in time efficiency, ergonomic and ecologic benefits are examined. However, specific limits of using virtual reality tools are being tested too. Even if the implementation of augmented reality in the industry is one of the most promising technologies in the context of Industry 4.0 - the implementation is still challenging because specific standards are missing [1].

The benefits of human-robot collaboration - though connected with costs and needs for mangers identify and eliminate potential hazards and erroneous behaviour - lie in the implementation of flexible and highly reconfigurable production system, which can easily change their operation to accommodate different product families, similar to the way that a human operator would do. In an attempt to follow the requirements for the more customized products, "new forms of between robots and humans aim to make the most out of the synergy effect to efficiently combine and exploit the robots' precision, repeatability, and strength with the human's intelligence and flexibility [17]. According to the authors, "such attributes are 
indispensable when considering the sustainability of small and medium-sized (SMEs) production firms who have to bear the costs for re-equipping or modifying their current production systems. Reducing the physical burden and risk of human-robot collaboration, the question of safety needs to be examined fully yet and it belongs to the main challenges for future.

\section{DISCUSSION}

Working with robots without usual safety areas requires to follow how (and to what degree) a specific design and integration of natural user interfaces could enhance the acceptance of humanoid robotic design in hybrid working teams. Different production environments (industry, service) seem to relate to different robotic design, as stated by [5], as well as different types of personalities are correlated with different types of reaction to a robot as a working partner. When comparing the humanoid and robotic design of robot in the context of different reactions of humans within a human-robot collaboration, empirical results have confirmed more stable reaction of different personality types to humanoid robot (false, mistaken) reactions, while reactions to robots false and mistaken behaviour have been found depending on the type of personality [6]. Different personality types, as well as the attitudes towards human-robot collaboration, seem to act as the factors of developing successful human-robot collaboration. However, people collaborate best with a proactive robot, but they prefer having control of when the robot should help, rather than working with a reactive robot that only helps when needed [13]. Studying interaction of specific personalities in the context of Human-Robot collaboration can support decisions for working in hybrid work teams.

New moderators and new determinants have been included in the UTAUT/2 model when studying technology adoption. However, also in the basic moderators (as gender, age), the Industry 4.0 innovation opens space for new examinations owing to the new technical means of Internet of Things. According to Scholtz [4], studies have shown that the ability to perceive information, integrate pieces of information and forecast future situation events (situational awareness) decreases with the age. The researchers can focus the attention on age-related differences or personality-related differences in our ability to perceive important information while working in mixed human-robot work teams and while working in virtual work environments. For example, in the frame of Industry 4.0 innovation: could technical means such as virtual reality glasses help in compensating the decrease in perceiving the information skills of workers related to their age.

\section{CONCLUSION}

According to the introduced UTAUT model of technology adoption, several core determinants of interaction and usage (performance expectancy, effort expectancy, social influence, and facilitating conditions) and up to four core determinants of key relationships (gender, age, experience, voluntariness of use) can be identified [14].

Working in virtual reality offers a new field of human capital development analysis. New questions arise as regard to combining real and virtual training settings to optimize the training and learning process. It can be supposed that specific models of technology adoption - as UTAUT/UTAUT2 - will require new extensions in a context of work analysis in a virtual work environment, e.g. health and psychological assumptions.

Based on the literature review of the UTAUT model extensions, new endogenous factors can be included in the models of technology adoption and use, namely emotional attitude (trust towards technology, perceived threat) and new moderators as a location of organization and type of work-place based training in an organization. Similarly, new technological means of 
Industry 4.0 (virtual glasses) can help to raise the ability to acquire important information (in a context of situational awareness) while working within human-robot mixed work teams and virtual work environments.

Regarding the findings in human-robot collaboration by Richert et al. [6], we suggest including the type of personality (the attitude towards Human-Robot collaboration) among the factors of technology adoption within the Industry 4.0 strategy. Emotional attachment acts as a relevant factor of successful Human-Robot collaboration that can be maintained by a support of team identification and self-extension process. For development of positive attitudes in human-robot collaboration, the principles of personalized design, e.g. engaging the workers in designing specific features of the robot, should be applied. One possibility to improve willingness to HRI and to increase the HRI acceptance is a mental simulation of both cooperative as well as competitive contact with a robot [19].

Within the Industry 4.0 concept, innovative tools within virtual and augmented reality bring intuitive and easy to see applications to communicate in human-robot collaboration with its benefits in time efficiency, ergonomic and ecologic benefits. However, specific limits of using the virtual reality tools are being tested too, and the implementation is still challenging since specific standards are missing [20].

Safety and well-being of workers, protection of health, willingness to cooperate within Human-Robot work teams and in virtual reality need to be considered comprehensively when analysing sustainable and successful adoption of the Industry 4.0 strategy.

Sustainable Human-Robot Interaction (SHRI) is as an innovative field of research and design [21] regarding the issues of Industry 4.0. We agree with authors Gubrod and Wiele [22] that sustainability covers all aspects that potentially impact the use of any limited resource. Then, sustainable HRI focuses on collaboration between humans and technology in the context of sustainability. New models originate to address this complex issues as evaluation of technology acceptance and sustainability during the technology life cycle, and forecast chances of technology to attract users in HRI, as e.g. the Integrated Acceptance and Sustainability Assessment Model [23, 24].

According to Venkatech et al. (2016), not many studies have paid attention to new types of the outcomes of the Industry 4.0 technology adoption within the UTAUT/UTAUT 2 model [25]. Subjective assessment of the increase in well-being of the workers after the adoption of the Industry 4.0 technologies can be identified as one of the new Industry 4.0 outcomes within the human capital definition of Industry 4.0.

\section{Acknowledgment}

This research was supported by the VEGA research Project [grant No. 2/0077/19] Work competencies in the context of Industry 4.0 development.

\section{References}

[1] ASKARPOUR, M., MANDRIOLI, D., ROSSI, M., VINCENTINI, F. 2019. Formal model of human errorneous behavior for safety analyses in collaborative robotics. In Robotics and computerintegrated Manufacturing, 57, pp. 456-476, ISSN: 0736-5845

[2] SCHOLTZ, J. 2002. Theory and Evaluation of Human Robot Interactions. Proceedings of the $36^{\text {th }}$ Hawaii International Conference in System Sciences. ISBN 978-0-7695-1874-9

[3] SOUSA, S., LAMAS D., DIAS P. 2014. A Model for Human-Computer Trust. In Zaphiris P., Ioannou A. (eds) Learning and Collaboration Technologies. Designing and Developing Novel Learning Experiences. LCT 2014. Lecture Notes in Computer Science, Vol. 8523. Springer, Cham, Online ISBN 978-3-319-07482-5

[4] KOVACS, G.; KOT, S. 2016. New Logistics and Production Trends as the Effect of Global Economy Changes. Pol. J. Manag. Stud., 14, pp.115-126. 
[5] MASLARIĆ, M., NIKOLIČIĆ, S., MIRČETIĆ, D. 2016. Logistics Response to the Industry 4.0: the Physical Internet. Open Engineering, (1). Online ISSN 2391-5439

[6] RICHERT, A., MÜLLER, S., SCHRÖDER, S., JESCHKE, S. 2018. Anthropomorphism in social robotics: empirical results on human-robot interaction in hybrid production workplaces. AI \&amp; Soc (2018) 33, p. 413-424. ISSN 0951-5666

[7] FIDLEROVÁ H., PORUBČINOVÁ M., FERO M., NOVOTNÁ I. 2019. Identification of Challenges and Opportunities for Work 4.0 Competences Developing in Slovakia In Human Capital Formation for the Fourth Industrial Revolution. IGI Global, pp. 44-72, DOI: 10.4018/9781-5225-9810-7.ch003, ISBN: 1522598103

[8] YOU, S., ROBER, L. 2008. Emotional Attachment, Performance, and Viability in Teams Collaborating with Embodied Physical Action (EPA) Robots. Journal of the Association for Information Systems, 19(5), 377-407. ISSN 1536-9323

[9] LEONHARD, G. 2016. Technology versus humanity Technology vs. Humanity: The coming clash between man and machine (FutureScapes). Kindle Edition, Fast Future Publishing, 186 p., ISBN10: 0993295827

[10] SHERIDAN, T. B. 2016. Human-Robot Interaction: Status and Challenges. Human Factors: The Journal of the Human Factors and Ergonomics Society, 58(4), pp. 525-532. https://doi.org/10.1177/0018720816644364

[11] PETERS, D., CALVO, R., A., RYAN, M. 2018. Designing for Motivation, Engagement and Wellbeing in Digital Experience. Frontiers in Psychology, 9(797), pp. 1-15. ISSN 1664-1078

[12] GROOM, V., NASS, C. 2007. Can robots be teammates? Benchmarks in human-robot teams. Interaction Studies, 8(3), pp. 483-500, ISSN 1572-0373

[13] BARAGLIA, J., CAKMAK, M., NAGAI, Y., RAO, R. P., \& ASADA, M. 2017. Efficient humanrobot collaboration: When should a robot take initiative? The International Journal of Robotics Research, 36(5-7), pp. 563-579. https://doi.org/10.1177/0278364916688253

[14] TURKLE, S. 2011. Alone Together: Why We Expect More from Technology and Less from Each Other. Hardcover. ISBN-10: 0465031463

[15] VENKATESH, V., MORRIS, M.G., DAVIS G.D., DAVIS. F.D. 2003. User Acceptance of IT. Individual reactions to using information technology. Intentions to use. MIS Quarterly, 27(3), 427. ISSN 2162-9730

[16] VENKATESH V., MORRIS. M.G. 2000. Age differences in technology adoption decisions. Implications for changing work force. In Personnel Psychology, 53, pp. 375-403. ISSN 1744-6570

[17] SCHULER, D., NAMIOKA, A. 1993. Participatory design: Principles and practices. Lawrence ERLBAUM ASSOCIATES, INC. ISBN 10: 0805809511

[18] MICHALOS, G., KOUSI, N., KARAGIANNIS, P.M GHOURNELOS, CH. 2018. SEAMLESS HUMAN robot collaboration assembly - An automotive case study. Mechatronics, Vol, 55, pp. 194-211. ISSN 0957-4158

[19] SATO, H. KAMIDE, Y., M. KOJIMA, M., ARAI, T. 2018. Friendly Motion Learning towards Sustainable Human Robot Interaction. IEEE/RSJ International Conference on Intelligent Robots and Systems (IROS), Madrid, pp. 848-853. ISSN 2153-0858

[20] FIORENTINO, M., UVA, A., GATTUllO, M., DEBERNARDIS, S., MONNO, G. 2014. Augmented reality on large screen for interactive maintenance instructions. Computers in Industry, 65(2), pp. 270-278. ISSN 0166-3615

[21] KUCHENBRANDT, D., EYSSEL, F. 2012. The mental simulation of a human-robot interaction: Positive effects on attitudes and anxiety toward robots. In 2012 IEEE RO-MAN: The 21st IEEE International Symposium on Robot and Human Interactive Communication, Paris, pp. 463-468, doi: 10.1109/ROMAN.2012.6343795.

[22] GUDBROD, R., WIELE, CH. 2012. The Software Dilemma: Balancing Creativity and Control on the Path to Sustainable Software. Berlin: Springer, p. 281.

[23] AIZSTRAUTA D., GINTERS E. Introducing Integrated Acceptance and Sustainability Assessment of Technologies: a Model based on System Dynamics Simulation. Springer LNBIP 145 Series "Modelling and Simulation in Engineering, Economics and Management", Springer LNBIP 145 Series "Modelling and Simulation in Engineering, Economics and Management". 
[24] AIZSTRAUTA, D., CELMINA, A., GINTERS, E., \& MAZZA, R. 2013. Validation of Integrated Acceptance and Sustainability Assessment Methodology. Procedia Computer Science, 26, 33-40. https://doi.org/10.1016/j.procs.2013.12.005

[25] VENKATESH, V., THONG, J., XUX. 2016. Unified Theory of Acceptance and Use of Technology: A Synthesis and the Road Ahead. Journal of the Association for Information Systems 17(5), pp. 328-376.

\section{ORCID}

Helena Fidlerová 0000-0002-3426-5803 